\title{
Biogeography based Optimization for Gain Maximization of Nine-Element Yagi-Uda Antenna
}

\author{
Ruchi Kansal \\ Yadvindra College of Engineering \\ Talwandi Saboo, Punjab [INDIA]
}

\author{
Ashwani Singla \\ Yadvindra College of Engineering \\ Talwandi Saboo, Punjab [INDIA]
}

\author{
Gagan Sachdeva \\ Rayat Bahra Group of Institutes \\ Mohali campus, Punjab [INDIA]
}

\begin{abstract}
Biogeography-Based Optimization (BBO) is a recently introduced population based algorithms which has shown impressive performance over other Evolutionary Algorithms (EAs). BBO is based on the study of distribution of biological organisms over space and time. BBO is a stochastic optimization technique, here, solutions for problem are considered as habitats whereas feature sharing, i.e. Suitability Index Variables (SIVs), among the habitats is known as migration and exploration of new SIV is accomplished as mutation. Yagi-Uda antenna design is most widely used antenna at VHF and UHF frequencies due to high gain, directivity and ease of construction. However, designing a Yagi-Uda antenna, that involves determination of optimal wire-lengths and their spacings, is a highly complex and non-linear engineering problem. In this paper, BBO algorithm is applied to optimize the lengths and spacings of nine-element Yagi-Uda antenna for maximum gain. The results obtained with this optimization technique are compared and the best results are tabulated in the ending sections of the paper.
\end{abstract}

\section{Keywords:}

Bio-geography Based Optimization (BBO), Particle Swarm Optimization (PSO), Genetic Algorithm (GA), Yagi-Uda Antenna, Antenna Gain, Antenna Impedance

\section{INTRODUCTION}

Antenna is an electrical device which forms an interface between free-space radiations and transmitter or receiver. The choice of an antenna depends on various factors such as gain, impedance, bandwidth, frequency of operation, sidelobe level (SLL), etc. A Yagi-Uda antenna is a widely used antenna design due to high forward gain capability, low cost and ease of construction. It is a parasitic linear array of parallel dipoles, one of which is energized directly by transmission line while others act as a parasitic radiators whose currents are induced by mutual coupling. The characteristics of Yagi-Uda antenna are affected by its geometric parameters. It is simple to construct and has a high gain, typically greater than $10 \mathrm{~dB}$ at $\mathrm{HF}$ and $\mathrm{UHF}$, i.e., $3 \mathrm{MHz}$ to $3 \mathrm{GHz}$.

A Yagi-Uda antenna was invented in 1926 by H. Yagi and S. Uda at Tohoku University [23] in Japan, however, published in English in 1928 [27]. Since its invention, continuous efforts have been put in optimizing the antenna for gain, impedance, SLL and bandwidth using different optimization techniques based on traditional mathematical approaches [17, 3, 7, 18, 6, 5, 8] and modern heuristic of Artificial Intelligence (AI) techniques [12, 26, 25, 2, 14, 22, 21]. In 1949, Fishenden and Wiblin [11] proposed an approximate design of Yagi aerials for maximum gain. In 1959, Ehrenspeck and Poehler have given a manual ap- proach to maximize the gain of the antenna by varying various lengths and spacings of its elements [10].

Later on, with the availability of computer software at affordable prices made it possible to optimize antennas numerically. Bojsen et al. [3] proposed another optimization technique to calculate the maximum gain of Yagi-Uda antenna arrays with equal and unequal spacings between adjoining elements. Cheng et al. [6, 5] have used optimum spacings and lengths to optimize the gain of a Yagi-Uda antenna. In [8], Cheng has proposed optimum design of Yagi-Uda antenna where antenna gain function is highly nonlinear.

In 1975, John Holland introduced Genetic Algorithms (GAs) as a stochastic swarm based AI technique, inspired from natural evolution of species, to optimize arbitrary systems for certain cost function. Many researchers, since then, investigated GAs to optimize Yagi-Uda antenna designs for gain, impedance and bandwidth separately [1, 12, 9] and collectively [26, 24, 13]. Baskar et $a l$. , in [2], have optimized Yagi-Uda antenna using Comprehensive Learning Particle Swarm Optimization (CLPSO) and presented better results than other optimization techniques. Li in [14] has used Differential Evolution (DE) to optimize geometrical parameters of a Yagi-Uda antenna and illustrated capabilities of the proposed method with several Yagi-Uda antenna designs. In [22], Singh et al. have explored another useful, stochastic global search and optimization technique named as Simulated Annealing (SA) for optimal Yagi-Uda antenna design.

In 2008, Dan Simon introduced yet another swarm based stochastic optimization technique based on science of biogeography where features sharing among various habitats, i.e., potential solutions, is accomplished with migration operator and exploration of new features is done with mutation operator [19]. Singh et al. have explored BBO as a better optimization technique for Yagi-Uda antenna designs, in [21].

In this paper, $\mathrm{BBO}$ algorithm are investigated to optimize the wire lengths of nine-element Yagi-Uda antenna and spacings in between them. A method of moments based programme, NEC2 (Numerical Electromagnetics Code version 2), is used to evaluate the antenna designs for gain, input impedance, bandwidth and beamwidth, etc.

After this brief literature review, the paper is outlined as follows: In the Section 2, Yagi-Uda antenna design parameters are discussed. Section 3 is dedicated to BBO algorithm. In Section 4, comparative simulation results for BBO are presented. Finally, conclusions and future scope have been discussed in Section 5.

\section{THE ANTENNA DESIGN PARAMETERS}

Usually, Yagi-Uda antenna consists of three types of elements: (a) Reflector-biggest among all and is responsible for blocking 
radiations in one direction. (b) Feeder-which is fed with the signal from transmission line to be transmitted and (c) Directors-these are usually more then one in number and responsible of unidirectional radiations. Designing a Yagi-Uda antenna involves determination of wire-lengths and wire-spacings in between to get maximum gain, desired impedance and minimum SLL at an arbitrary frequency of operation. An antenna with $N$ elements requires $2 N-1$ parameters, i.e., $N$ wire lengths and $N-1$ spacings, that are to be determined. These $2 N-1$ parameters, collectively, are represented as a string referred as a habitat in $\mathrm{BBO}$ given as (1).

$$
H=\left[L_{1}, L_{2}, \ldots, L_{N}, S_{1}, S_{2}, \ldots, S_{N-1}\right]
$$

where $L_{s}$ are the lengths and $S_{s}$ are the spacing of antenna elements. An incoming field sets up resonant currents on all the antenna elements which re-radiate signals. These re-radiated signals are then picked up by the feeder element, that leads to total current induced in the feeder equivalent to combination of the direct field input and the re-radiated contributions from the director and reflector elements. This makes highly non-linear and complex relationships between antenna parameters and its characteristics like gain, impedance and SLL, etc.

Figure 1 depicts a typical Yagi-Uda antenna where all wires are placed parallel to $X$-axis and along $Y$-axis. Middle segment of the reflector element is placed at origin and excitation is applied to the middle segment of the feeder element.

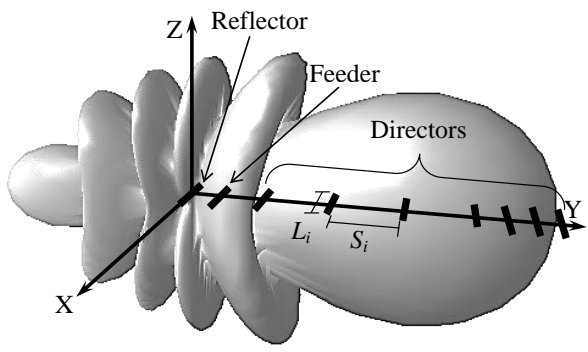

Fig. 1. Yagi-Uda Antenna

\section{BIO-GEOGRAPHY BASED OPTIMIZATION}

As name suggests, $\mathrm{BBO}$ is a population based global optimization technique developed on the basis of the science of biogeography, i.e., study of the distribution of animals and plants among different habitats over time and space. BBO results presented by researchers are better than other EAs [12, 24, 2, 16].

Mathematical models of biogeography describe migration, speciation, and extinction of species in various islands [15]. The term island is used for any habitat that is geographically isolated from other habitats. Habitats that are well suited residences for biological species are referred to have high High Suitability Index (HSI) similar to fitness in other EAs. However, HSI value of a habitat depends upon many factors such as rainfall, diversity of vegetation, diversity of topographic features, land area, and temperature, etc. The variables that characterize habitability are termed as Suitability Index Variables (SIVs). The habitats with a high HSI tend to have a large population of its resident species, that is responsible for more probability of emigration and less probability of immigration due to natural random behavior of species. Immigration is the arrival of new species into a habitat or population, while emigration is the act of leaving one's native region.

On the other hand, habitats with low HSI tend to have low emigration rate, $\mu$, due to sparse population, however, they will have high immigration rate, $\lambda$. Suitability of habitats with low HSI is likely to increase with influx of species from other habitats having high HSI. However, if HSI does not increase and remains low, species in that habitat go extinct that leads to additional immigration. For sake of simplicity, it is safe to assume a linear relationship between emigration rates and immigration rates. Additionally, same maximum emigration and immigration rates, i.e., $E=I$, as depicted graphically in Figure 2. For HSI of $k$ th habitat emigration and immigration rates are given by (2) and (3).

$$
\begin{gathered}
\mu_{k}=E \cdot \frac{H S I_{k}}{H S I_{\max }-H S I_{\min }} \\
\lambda_{k}=I \cdot\left(1-\frac{H S I_{k}}{H S I_{\max }-H S I_{\min }}\right)
\end{gathered}
$$

The immigration of new species from high HSI to low HSI habitats may raise the HSI of poor habitats as good solutions have more resistance to change than poor solutions whereas poor solutions are more dynamic and accept a lot of new features from good solutions.

A habitat in BBO is represented by $M$-dimensional vector as $H=\left[S I V_{1}, S I V_{2}, \ldots \ldots \ldots, S I V_{M}\right]$, called habitat, where $M$ is the number of SIVs to be evolved for optimal HSI. HSI is the degree of acceptability determined by the fitness function associated with each habitat in a population of $N P$ habitats. Following subsections discuss two constituents operators of BBO, viz. migration and mutation:

\subsection{Migration}

Migration is a probabilistic operator that improves HSI of poor habitats by sharing features from good habitats. During Migration, immigrating habitat, ImHbt, use its immigration rate, $\lambda$, given by (3), to probabilistically decide whether to immigrate or not. In case immigration is selected, then the emigrating habitat, EmHbt, is found probabilistically based on emigration rate, $\mu$, given by (2). The process of migration is completed by copying values of SIVs from EmHbt to ImHbt at random chosen sites. This is termed as the standard migration operator. Generalized Pseudo Code for Migration

for $i=1$ to $N P$ do

Select $H_{i}$ with probability based on $\lambda_{i}$

if $H_{i}$ is selected then

for $j=1$ to $N P$ do

Select $H_{j}$ with probability based on $\mu_{j}$

if $H_{j}$ is selected

Randomly select a $\operatorname{SIV}(s)$ from $H_{j}$

Copy them $\operatorname{SIV}(s)$ in $H_{i}$

end if

end for

end if

end for

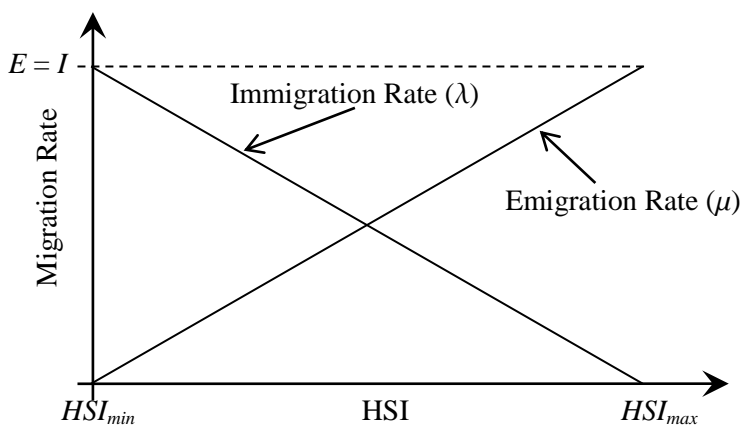

Fig. 2. Migration Curves 


\subsection{Mutation}

Mutation is probabilistic operator that randomly modifies the values of some randomly selected SIVs that is intended for exploration of search space for better solutions by increasing the biological diversity in the population. Here, increased mutation rate is investigated on habitats those are, probabilistically, participating less in migration. The mutation rate, mRate, for $i$-th habitats is calculated as (4)

$$
\text { mRate }=C \cdot \min \left(\mu_{k}, \lambda_{k}\right)
$$

where $C$ is a constant and equal to 1 . The pseudo code for standard mutation operator is depicted in Algorithm 3.2. In our simulations, like other population-based optimization techniques, elitism is considered so as to preserve best solution from one generation to next. Generalized Pseudo Code for Mutation

$$
\text { mRate }=C \times \min \left(\mu_{k}, \lambda_{k}\right) \text { where } C=1
$$

$$
\text { for } i=1 \text { to } N P \text { do }
$$

$$
\text { for } j=1 \text { to length }(H) \text { do }
$$

Select $H_{j}(\mathrm{SIV})$ with mRate

If $H_{j}(\mathrm{SIV})$ is selected then

Replace $H_{j}(\mathrm{SIV})$ with randomly generated SIV

$$
\text { end for }
$$$$
\text { end i }
$$

end for

\section{SIMULATION RESULTS AND DISCUSSIONS}

Nine-wire Yagi-Uda antenna designs are optimized for gain using $\mathrm{BBO}$, discussed in Section 3, are investigated along with standard mutation operator. To present fair analysis, design is optimized with 30 habitats using 200 iterations. The $\mathrm{C}++$ programming platform is used for coding of $\mathrm{BBO}$ algorithm, whereas, a NEC2 [4] is used for evaluation of antenna designs based on method of moments. Each potential solution in BBO is encoded as vector with 17 SIVs as given by (1). The radiation characteristics of Yagi-Uda antenna can change significantly by varying element lengths and spacings upto four decimal places. The universe of discourse for the search of optimum values of wire lengths and wire spacings are $0.40 \lambda-0.50 \lambda$ and $0.10 \lambda-0.50 \lambda$, respectively, however, cross sectional radius and segment sizes are kept same for all elements, i.e., $0.003397 \lambda$ and $0.1 \lambda$ respectively, where $\lambda$ is the wavelength corresponding to frequency of operation, i.e, $300 \mathrm{MHz}$. Excitation is applied to the middle segment of driven element and location of middle segment of the reflector element is always kept at $x=0$. The scaling constant $C$, the maximum migration rates $E$ and $I$, are set equal to 1 . The

\begin{tabular}{|c|c|c|c|c|c|c|}
\hline \multirow[b]{2}{*}{ Element } & \multicolumn{2}{|c|}{ Six-Element [21] } & \multicolumn{2}{|c|}{ Six-Element [20] } & \multicolumn{2}{|c|}{ Nine-Element } \\
\hline & Length & Spacing & Length & Spacing & Length & Spacing \\
\hline $1(\lambda)$ & 0.4838 & - & 0.4872 & - & 0.4796 & - \\
\hline $2(\lambda)$ & 0.4690 & 0.1722 & 0.4947 & 0.1540 & 0.4539 & 0.2658 \\
\hline $3(\lambda)$ & 0.4450 & 0.2193 & 0.4407 & 0.2554 & 0.4424 & 0.2007 \\
\hline $4(\lambda)$ & 0.4226 & 0.4406 & 0.4244 & 0.4001 & 0.4206 & 0.4245 \\
\hline $5(\lambda)$ & 0.4218 & 0.3954 & 0.4217 & 0.4048 & 0.4170 & 0.4261 \\
\hline $6(\lambda)$ & 0.4286 & 0.3754 & 0.4277 & 0.3775 & 0.4146 & 0.4002 \\
\hline $7(\lambda)$ & - & - & - & - & 0.4108 & 0.4592 \\
\hline $8(\lambda)$ & - & - & - & - & 0.4174 & 0.3923 \\
\hline $9(\lambda)$ & - & - & - & - & 0.4159 & 0.4546 \\
\hline Gain(dBi) & \multicolumn{2}{|c|}{13.84} & \multicolumn{2}{|c|}{13.85} & \multicolumn{2}{|c|}{15.89} \\
\hline $\mathrm{Z}(\Omega)$ & \multicolumn{2}{|c|}{$3.229+\mathrm{j} 23.42$} & \multicolumn{2}{|c|}{$4.45+\mathrm{j} 63.20$} & \multicolumn{2}{|c|}{$4.68+\mathrm{j} 1.80$} \\
\hline
\end{tabular}
best antenna designs obtained during process of design optimization of nine-element Yagi-Uda antenna are tabulated in Table 1.

Table 1. The best antenna designs obtained during optimization

It can be seen from Table 1 that maximum gain of $15.89 \mathrm{dBi}$ of nine-element Yagi-Uda antenna obtained with BBO, whereas,
$13.84 \mathrm{dBi}[21]$ and $13.85 \mathrm{dBi}[20]$ gain of six-element Yagi-Uda antenna has obtained using same optimization technique. To the best literature available, gain obtained by BBO, i.e., $15.89 \mathrm{dBi}$ is the highest gain that obtained from nine-element Yagi-Uda antenna yet.

\section{CONCLUSIONS AND FUTURE SCOPE}

In this paper, BBO algorithm is applied to optimize nine-element Yagi-Uda antenna for gain maximization. As per literature review, the gain obtained with BBO is the maximum gain of nineelement Yagi-Uda antenna. Investigation of variants of PSO and BBO algorithms for improved performance of Yagi-Uda antenna and for other types of antenna designs is next on our agenda.

\section{REFERENCES}

[1] E.E. Altshuler and D.S. Linden. Wire-antenna Designs using Genetic Algorithms. Antennas and Propagation Magazine, IEEE, 39(2):33-43, 1997.

[2] S. Baskar, A. Alphones, P N Suganthan, and J J Liang. Design of Yagi-Uda Antennas using Comprehensive Learning Particle Swarm Optimisation. IEEE, 152(5):340-346, 2005.

[3] JH Bojsen, H. Schjaer-Jacobsen, E. Nilsson, and J. Bach Andersen. Maximum Gain of Yagi-Uda Arrays. Electronics Letters, 7(18):531-532, 1971.

[4] G. J. Burke and A. J. Poggio. Numerical Electromagnetics Code (NEC) method of moments. NOSC Tech. DocLawrence Livermore National Laboratory, Livermore, Calif, USA, 116:1-131, 1981.

[5] C. Chen and D. Cheng. Optimum Element Lengths for Yagi-Uda Arrays. IEEE Transactions on Antennas and Propagation, , 23(1):8-15, 1975.

[6] D. Cheng and C. Chen. Optimum Element Spacings for Yagi-Uda Arrays. IEEE Transactions on Antennas and Propagation, 21(5):615-623, 1973.

[7] D. K. Cheng. Optimization Techniques for Antenna Arrays. Proceedings of the IEEE, 59(12):1664-1674, 1971.

[8] D. K. Cheng. Gain Optimization for Yagi-Uda Arrays. Antennas and Propagation Magazine, IEEE, 33(3):42-46, 1991.

[9] D. Correia, A. J. M. Soares, and M. A. B. Terada. Optimization of gain, impedance and bandwidth in Yagi-Uda Antennas using Genetic Algorithm. IEEE, 1:41-44, 1999.

[10] H. Ehrenspeck and H. Poehler. A New Method for Obtaining Maximum Gain from Yagi Antennas. IRE Transactions on Antennas and Propagation, , 7(4):379-386, 1959.

[11] R. M. Fishenden and E. R. Wiblin. Design of Yagi Aerials. Proceedings of the IEE-Part III: Radio and Communication Engineering, 96(39):5, 1949.

[12] E. A. Jones and W. T. Joines. Design of Yagi-Uda Antennas using Genetic Algorithms. IEEE Transactions on Antennas and Propagation, 45(9):1386-1392, 1997.

[13] Y. Kuwahara. Multiobjective Optimization Design of YagiUda Antenna. IEEE Transactions on Antennas and Propagation, 53(6):1984-1992, 2005.

[14] J. Y. Li. Optimizing Design of Antenna using Differential Evolution. IEEE, 1:1-4, 2007

[15] R.H. MacArthur and E.O. Wilson. The Theory of Island Biogeography. Princeton Univ Pr, 1967.

[16] M. Rattan, M. S. Patterh, and B. S. Sohi. Optimization of Yagi-Uda Antenna using Simulated Annealing. Journal of Electromagnetic Waves and Applications, 22, 2(3):291299, 2008. 
[17] D. G. Reid. The Gain of an Idealized Yagi Array. Journal of the Institution of Electrical Engineers-Part IIIA: Radiolocation, , 93(3):564-566, 1946.

[18] L. C. Shen. Directivity and Bandwidth of Single-band and Double-band Yagi Arrays. IEEE Transactions on Antennas and Propagation, 20(6):778-780, 1972.

[19] D. Simon. Biogeography-based Optimization. IEEE Transactions on Evolutionary Computation, 12(6):702-713, 2008.

[20] Satvir Singh and Gagan Sachdeva. Mutation Effects on BBO Evolution in Optimizing Yagi-Uda Antenna Design. In IEEE, Emerging Applications of Information Technology (EAIT), 2012 Third International Conference on, pages 47-51. IEEE, 2012.

[21] U. Singh, H. Kumar, and T. S. Kamal. Design of Yagi-Uda Antenna Using Biogeography Based Optimization. IEEE Transactions on Antennas and Propagation, , 58(10):33753379, 2010.
[22] U. Singh, M. Rattan, N. Singh, and M. S. Patterh. Design of a Yagi-Uda Antenna by Simulated Annealing for Gain, Impedance and FBR. IEEE, 1:974-979, 2007.

[23] Shintaro Uda and Yasuto Mushiake. Yagi-Uda Antenna. Maruzen Company, Ltd, 1954

[24] N. V. Venkatarayalu and T. Ray. Single and Multi-Objective Design of Yagi-Uda Antennas using Computational Intelligence. IEEE, 2:1237-1242, 2003.

[25] N.V. Venkatarayalu and T. Ray. Optimum Design of YagiUda Antennas Using Computational Intelligence. IEEE Transactions on Antennas and Propagation,, 52(7):18111818, 2004.

[26] H. J. Wang, K. F. Man, C. H. Chan, and K. M. Luk. Optimization of Yagi array by Hierarchical Genetic Algorithms. IEEE, 1:91-94, 2003.

[27] H. Yagi. Beam Transmission of Ultra Short Waves. Proceedings of the Institute of Radio Engineers, 16(6):715740, 1928. 